\title{
Does it Matter How We Pose the Question "How is Your Sense of Smell?"
}

\author{
Eike Wehling • Astri J. Lundervold • Steven Nordin
}

Received: 9 December 2013 / Accepted: 11 July 2014 / Published online: 29 July 2014

(C) Springer Science+Business Media New York 2014

\begin{abstract}
There is a rather large, and unfortunate, discrepancy in the outcome between self-reported and standardized assessment of olfactory function. Questions for self-evaluation are commonly used that provide no information of with what to compare (comparison target) one's olfactory function. We therefore investigated whether responses differed between an unspecific question and two questions providing comparison targets. Ninety-six healthy community-dwelling individuals ( $62.5 \%$ women) aged $49-80$ years evaluated their odor identification ability, followed by standardized assessment of odor identification ability. Results revealed that response patterns varied significantly depending on comparison target. While $81 \%$ reported normal function when no further comparison target was presented, $69 \%$ reported normal function when referring to age-related olfactory changes in identification ability. In turn, sensitivity of the accuracy of self-reported reduced odor identification ability (with standardized assessment as reference) increased from 11 to $37 \%$, whereas specificity decreased from 86 to $71 \%$ when providing a comparison target. Accuracy of self-reported olfactory function can be increased by including a comparison target. However, standardized assessment is to be preferred over self-reported assessment, irrespective of how the question is formulated.
\end{abstract}

Keywords Olfaction · Aging · Self-report · Smell ·

Sensitivity $\cdot$ Specificity

\author{
E. Wehling $(\triangle) \cdot$ A. J. Lundervold \\ Department of Biological and Medical Psychology, University of \\ Bergen, Jonas Lies vei 91, 5009 Bergen, Norway \\ e-mail: eike.wehling@psybp.uib.no \\ E. Wehling $\cdot$ A. J. Lundervold \\ Kavli Research Centre for Aging and Dementia, Haraldsplass \\ Hospital, Bergen, Norway \\ S. Nordin \\ Department of Psychology, Umeå University, Umeå, Sweden
}

\section{Introduction}

The sense of smell is important as it is involved in digestion, warning of danger (e.g., fires or spoiled food), and social interaction. Its loss may reduce quality of life considerably (Croy et al. 2014). Yet, even a complete loss of the sense of smell may go unnoticed, and rather large discrepancies between subjective reports and test measures are common. In an epidemiologic study, Murphy et al. (2002) found in a sample aged 53-97 years that $9.4 \%$ reported impaired olfactory function whereas an objectively measured dysfunction by means of an odor identification task was shown in $24.5 \%$. These findings are confirmed by other studies of aging individuals showing low sensitivity (correctly reporting dysfunction) and high specificity (correctly reporting normosmia) of self-reported olfactory functioning (Nordin et al. (1995): sensitivity in healthy elderly controls $23 \%$, specificity $49 \%$; Wehling et al. (2011): sensitivity $19 \%$, specificity $91 \%$ ).

The reason for the discrepancy between subjective and objective measures is poorly understood. Landis et al. (2003) found that experience with olfactory assessment resulted in the participant more correctly evaluating the own olfactory status. This was in contrast to their finding showing that ratings of olfactory function in participants unexperienced with olfactory assessment correlated significantly with ratings of nasal airway resistance, but not with assessed function. The evaluation of the olfactory status appears to be more correct among ear-nose-throat patients seeking medical attention (Nordin et al. 1995; Welge-Luessen et al. 2005; Klimek et al. 1998; but see also White and Kurtz (2003)).

So far, little attention has been paid to the questions used to examine self-reports. Frequently, with some variation, a single question is asked, such as "How would you estimate your sense of smell?" Hence, the individual is requested to make a judgment although no information is given about a referred comparison dimension. A rather unspecific question of this 
kind may lead individuals in their 20s and those in their 70 s to consider their function as normal, as they tend to compare themselves with similar others in their social environment (Wood 1989). Thus, a somewhat reduced sense of smell found in older age may be considered as "normal" by the respondent in spite of impairment according to test results.

Alternatively, an individual may use a temporal comparison target by relating his/her present olfactory function to that in the past. Two recent studies examined this issue, requesting participants to indicate if they had experienced changes in their olfactory function (Bahar-Fuchs et al. 2011; Djordjevic et al. 2008). None of the studies found significant correlations between self-reports and assessed function. However, BaharFuchs et al. (2011) commented that their question on changes during the past 12 months may have been a rather short interval so that gradual changes may have gone unnoticed.

Thus, in the process of self-reporting olfactory function, it is typically unclear whether a social comparison appraisal (comparing function with that of others, and if so with whom) or a temporal comparison appraisal (comparing current function with that of the past) is to be applied. This motivated the present study to examine whether response patterns in selfreports vary depending on reference group presented in the question In a final step, sensitivity and specificity of selfreported odor identification ability were examined, combining self-reported function with results from a standardized odor identification task.

\section{Methods and Materials}

\section{Participants}

Participants were part of a longitudinal study on cognitive aging. Recruitment was carried out through advertisement in local newspapers. All participants were native speakers of Norwegian, had completed obligatory basic education, were living independently at home by the time of assessment, and did not report current or past neurological or psychiatric disorders or head trauma. All participants gave their informed consent, and the project was approved by the Regional Committee for Research Ethics of Southern Norway. The sample of this study consisted of 96 participants after excluding two participants who were classified as anosmic on the odor detection screening task (see below) and 21 who did not complete the olfactory questionnaire. Characteristics of the final sample are given in Table 1.

\section{Self-Reports on Olfactory Functioning}

The participants underwent initially a short questionnaire regarding factors with potential effect on olfactory functioning
Table 1 Sample characteristics and self-reported factors with potential effect on olfactory performance

\begin{tabular}{lll}
\hline & & Range \\
\hline Gender (males/females) & $34 / 62$ & \\
Age (years) & $63.8(7.6)^{\mathrm{a}}$ & $49-80$ \\
Education (years) & $13.9(2.9)^{\mathrm{a}}$ & $8-20$ \\
MMSE (Mini Mental State & $28.9(1.0)^{\mathrm{a}}$ & $26-30$ \\
$\quad$ Examinantion; Folstein et al. 1975) & & \\
Problems breathing through the nose $(n)$ & 14 & \\
Polyposis (n) & 3 & \\
Smoking $(n)$ & 5 & \\
Olfactory status (SOIT) & $69 / 24 / 3$ & \\
$\quad$ (normosmic/hyposmic/anosmic) $(n)$ & & \\
\hline
\end{tabular}

${ }^{\mathrm{a}}$ Mean (standard deviation)

(Table 1). This was followed by three questions, regarding self-reported identification ability (Table 2 ). While the first question did not refer to a specific reference group, the other two referred to specific comparison targets. Response categories were either (1) worse than (the reference group as indicated in the question) or (2) the same as or better than (the reference group as indicated in the question).

\section{Olfactory Assessment}

The participants first answered the questions regarding olfactory functioning, followed by a detection sensitivity screening and the assessment according to an odor identification test, the Scandinavian Odor Identification Test (SOIT).

\section{Odor Detection Sensitivity}

Smell dysfunction may result from several causes (see Seiberling and Conley (2004); Murphy et al. (2003), for an overview). Since the overall project design has its focus on healthy cognitive aging, we wanted to assure inclusion of participants who had at least some remaining odor detection sensitivity. Therefore, all participants were assessed with a simplified version of the Connecticut Chemosensory Clinical Research Center Threshold Test (Cain 1989) to screen for anosmia. A two-alternative, forced-choice ascending method of limits was used with two concentrations of butanol dissolved in deionized water, 0.58 and $336 \mathrm{ppm}$. Starting with the weakest concentration, the participant was presented with the odorant and a blank solution in randomized order and asked to indicate which of the two presentations smelled stronger. An incorrect choice on any trial (choosing the blank solution) led to an 
Table 2 Questions about identification ability with varying comparison targets

How would you evaluate your ability to identify odors compared to... Answer categories

\begin{tabular}{|c|c|c|c|}
\hline No further comparison target presented & Worse than normal & Normal & Better than normal \\
\hline Individuals at your age & Worse than individuals at my age & Same & Better than individuals at my age \\
\hline Yourself at a younger age (when you were in your $30 \mathrm{~s}$ ) & Worse than when I was younger & Same & Better than when I was younger \\
\hline
\end{tabular}

increase in concentration, whereas a correct choice led to repeated presentation of the concentration until four consecutive correct choices for one concentration were given. Trials were approximately $90 \mathrm{~s}$ apart to avoid adaptation. No detection of $336 \mathrm{ppm}$ was defined as anosmia. The vapor-phase concentration of $n$-butanol was calculated according to Cometto-Muñiz et al. (2003). Two participants were classified as anosmic and were excluded from further assessment.

\section{Odor Identification}

The SOIT was used to assess olfactory function. The test has adequate psychometric properties (test-retest reliability $r=0.79$, validity $r=0.76$; Nordin et al. 1998) and includes 16 odorants: pine needle, peppermint, juniper berry, violet, anise, clove, vanilla, almond (bitter), orange, cinnamon, lemon, lilac, vinegar, tar, ammonia, and apple. Ammonia $(1.0 \mathrm{M})$, tar, and vinegar are natural products, while the other odorants are natural oils (Stockholm Ether \& Essence Manufactory, Stockholm, Sweden). The odorant was injected into a tampon filled to saturation and placed into an opaque, 80-ml glass jar, sealed with a Teflon lock. The stimuli were presented birhinally $1-5 \mathrm{~cm}$ under the participants' nose. A card with four written response alternatives was placed in front of the participant with the instruction to choose the item that most appropriately identified the odor, with a forced-choice procedure. To avoid effects of adaptation, each stimulus was presented for 3-4 s and with an inter-stimulus interval of about 20 s (Cain and Engen 1969). No time restrictions were given for the participants to make their choice. Testing was conducted in a well-ventilated room without background odor. According to normative data, normosmia was defined as a sum of 13-16 correct answers, hyposmia as a sum of 10-12 correct answers (2 SDs below the mean), and anosmia as a sum of $\leq 9$ correct answers (4 SDs below the mean).

\section{Statistical Analyses}

Statistical analyses were performed using SPSS 20.0 (SPSS, Inc. Chicago, IL). Chi-square tests were used to compare frequencies, and $t$ tests to compare means. Descriptive statistics are presented as means and standard deviation. The level of significance was set at $p<0.05$.

\section{Results}

Self-Reports and Comparison Targets

Analyses revealed significant differences in pattern between self-reports depending on comparison targets $\left(\chi^{2}\right.$ $(2)=17.86, p<0.01$ ) (Fig. 1). Post hoc pairwise chisquare analyses revealed significant differences between all comparison groups (all $p<0.05$ ), but no significant differences in self-reports between either age groups or men and women.

\section{Accuracy of Self-Reported Odor Identification Ability}

The distribution of odor identification performance scores ranged from 8 to 16 (16 being the highest achievable score) and was slightly negatively skewed. Performance was significantly associated with age $(r=-0.23)$, and women outperformed men significantly (women M 13.98 \pm 1.6 ; men 12.71 $\pm 2.1 ; t(94)=-3.38$, $p=0.001$ ). According to normative data for the SOIT, $72 \%$ of the participants were classified as normosmic (58\% men), $25 \%$ as hyposmic (33\% men), and $3 \%$ as anosmic (all men) (Table 3). Three, seven, and ten percent of the participants (individuals at same age/ unspecific comparison target/yourself at a younger age, respectively) correctly reported reduced identification ability. None of the individuals performing on anosmia level reported reduced identification ability. The sensitivity of self-reported odor identification ability (correctly reporting reduced odor identification ability) varied between 11, 26, and $37 \%$ (individuals at same age/unspecific comparison target/yourself at a younger age, respectively), while the specificity of self-reported identification ability (correctly reporting normal odor identification ability) varied between 71 , 86 , and $94 \%$ (yourself at a younger age/unspecific 
Fig. 1 Percentage of participants reporting reduced/worse identification ability when reporting olfactory function depending on comparison targets

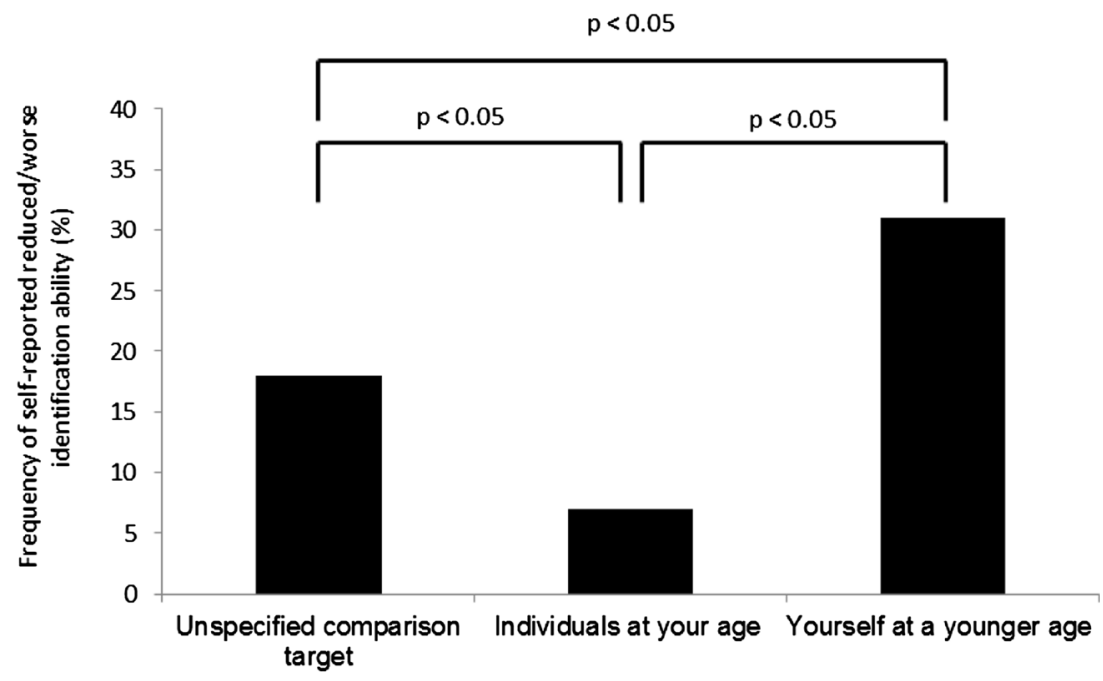

comparison target/individuals at the same age, respectively).

\section{Discussion}

The objective of the present study was to examine whether providing comparison targets in the question on olfactory function had an impact on self-reports of olfactory functioning. The results revealed that the availability of reference groups resulted in noteworthy patterns, showing that $31 \%$ of the participants reported age-related changes in identification ability. Thus, the sensitivity (correctly reporting reduced odor identification ability) increased when changes in olfactory function were addressed whereas specificity (correctly reporting normal identification ability) decreased.

The findings regarding self-reported olfactory function in comparison to specific targets are also noteworthy. The emerging pattern demonstrates two highly similar response patterns for the unspecific target and the socially prominent comparison target (individuals at your age), which contrast to the response pattern deriving from the temporal comparison target (yourself at a younger age).
The finding that a significant lower proportion of individuals reported their identification ability to be poorer/worse than individuals at the same age may be explained by findings showing that such reports may be in favor of one's own standing (Taylor and Brown 1988; Weinstein 1980). The findings showing that as many as $31 \%$ of the participants reported that their functional level was poorer compared to earlier in life are rather new. Nordin et al. (2004) reported that the prevalence of self-reported reduced detection sensitivity increased above $30 \%$ in aging individuals beyond the age of 70 years. The authors asked participants explicitly to report detection sensitivity (in reference to the general population). Earlier studies have reported lower numbers in comparable age groups (Murphy et al. (2002) $9.5 \%$; Karpa et al. (2010) $11 \%$; Bramersson et al. (2004) $14 \%$ ). Thus, as suggested by Bahar-Fuchs et al. (2011), it seems that a temporal comparison target may add information and possibly increase the correctness of self-reports.

In fact, the final analyses showed that sensitivity (correctly reporting reduced odor identification ability) was as high as $37 \%$ when self-reports on age-related changes (comparison target yourself at a younger age) were combined with results from an odor identification

Table 3 Concordance $(n)$ between self-reported and assessed odor identification ability

\begin{tabular}{|c|c|c|c|c|c|c|c|}
\hline \multirow[b]{3}{*}{ Measured function } & \multicolumn{7}{|c|}{ Self-reported odor identification ability } \\
\hline & \multicolumn{2}{|c|}{ Unspecific comparison target } & \multicolumn{2}{|c|}{ Individuals at your age } & \multicolumn{2}{|c|}{ Yourself at a younger age } & \multirow[t]{2}{*}{ Total } \\
\hline & Normal & Reduced & Same & Worse & Same & Worse & \\
\hline Normosmia & 59 & 10 & 65 & 4 & 49 & 20 & 69 \\
\hline Hyposmia/anosmia & 20 & 7 & 24 & 3 & 17 & 10 & 27 \\
\hline Total & 79 & 17 & 89 & 7 & 66 & 30 & 96 \\
\hline
\end{tabular}


task. This proportion is higher than earlier reported (Murphy et al. 2002; Nordin et al. 1995; Karpa et al. 2010) but still rather moderate. Yet, the positive increase in sensitivity came along with a decrease in specificity (correctly reporting normal identification ability). Although speculative, an explanation may be that the word "younger" "triggered" the common notion that age is taking its toll on all functions, including olfaction. We could not replicate the findings of Djordjevic et al. (2008) showing that anosmic participants correctly reported their dysfunction. This may be explained by the remaining sensitivity in our participants and that their dysfunction does not (yet) interfere with everyday life.

Our study has limitations in that the sample contained rather well-functioning adults, limiting the generalizability. However, it also shows that rather well-functioning adults may have difficulties to evaluate their sense of smell and that such difficulties are not restricted to cognitively impaired individuals.

Yet, despite these limitations, we consider our findings to be important as they address the problem of operationalization questions on olfactory functioning. Clinicians should be aware of the impact of comparison targets when formulating a question regarding olfactory functioning. In sum, our findings demonstrate that accuracy of self-reported olfactory functions may be increased in aging individuals when the question includes a temporal comparison target so that the respondent may include age-related changes. However, the concurrent drop in specificity demonstrates the trade-off coming along with this way of questioning.

In sum, we conclude that standardized assessment has to be preferred to the reliance on self-reported olfactory functioning, irrespective the way questions are formulated.

Acknowledgments This work was supported by grant 911461 from the Regional Health Authorities Bergen (Helse Bergen) to Eike Wehling. We would like to thank Benedicte Mjeldheim, Randi Hopsdal, and Nina Harkestad for the technical support.

\section{Compliance with Ethics Requirements}

Conflict of Interest Eike Wehling declares that she has no conflict of interest.

Astri J. Lundervold declares that she has no conflict of interest.

Steven Nordin declares that he has no conflict of interest.

All procedures followed were in accordance with the ethical standards of the responsible committee on human experimentation (institutional and national) and with the Helsinki Declaration of 1975, as revised in 2013. Informed consent was obtained from all patients for being included in the study.

\section{References}

Bahar-Fuchs A, Moss S, Rowe C, Savage G (2011) Awareness of olfactory deficits in healthy aging, amnestic cognitive impairment and Alzheimer's disease. Int Psychogeriatr 1-10
Bramersson A, Johansson L, Ek L, Nordin S, Bende M (2004) Prevalence of olfactory dysfunction: the Skovde population-based study. Laryngoscopy 114:1171-1173

Cain WS (1989) Testing olfaction in a clinical setting. Ear Nose Throat J 68:78-86

Cain WS, Engen T (1969) Olfactory adaptation and the scaling of odor intensity. In: Pfaffman C (ed) Olfaction and taste III. Rockefeller University Press, New York, pp 127-141

Cometto-Muñiz JE, Cain WS, Abraham MH (2003) Quantification of chemical vapors in chemosensory research. Chem Senses 28:467477

Croy I, Nordin S, Hummel T (2014) Olfactory disorders and quality of life - an updated review. Chem Senses 39:185-194

Djordjevic J, Jones-Gotman M, DeSousa K, Chertkow H (2008) Olfaction in patients with mild cognitive impairment and Alzheimer's disease. Neurobiol Aging 29:693-706

Folstein MF, Folstein SE, McHugh PR (1975) Mini-mental state- practical method for grading cognitive state of patients for clinician. $\mathrm{J}$ Psychiatry Res 12:189-198

Karpa MJ, Gopinath B, Rochtchina E, Wang, JJ Cumming RG, Sue CM, Mitchell P (2010) Prevalence and neurodegenerative or other associations with olfactory impairment in an older community. J Aging Health 22:154-168

Klimek L, Hummel T, Moll B, Kobal G, Mann WJ (1998) Lateralized and bilateral olfactory function in patients with chronic sinusitis compared with healthy control subjects. Laryngoscopy 108:111114

Landis BN, Hummel T, Hugentobler M, Giger R, Lacroix JS (2003) Ratings of overall olfactory function. Chem Senses 28:691-694

Murphy C, Schubert CR, Cruickshanks KJ, Klein BEK, Klein R, Nondahl DM (2002) Prevalence of olfactory impairment in older adults. JAMA 288:2307-2312

Murphy C, Doty RL, Duncan HJ (2003) Clinical disorders of olfaction. In: Doty RL (ed) Handbook of olfaction and taste, 2nd edn. Marcel Dekker, Inc., New York, pp 461-479

Nordin S, Monsch A, Murphy C (1995) Unawareness of smell loss in normal aging and Alzheimer's disease: discrepancy between self-reported and diagnosed smell sensitivity. J Gerontol 50B: $187-192$

Nordin S, Brämerson A, Lidén E, Bende M (1998) The Scandinavian Odor-Identification Test: development, reliability, validity, and normative data. Acta Otolaryngol 118:226-234

Nordin S, Brämerson A, Bende M (2004) Prevalence of self-reported poor odor detection sensitivity: the Skövde population-based study. Acta Otolaryngol 124:1171-1173

Seiberling KA, Conley DB (2004) Aging and olfaction and taste function. Otolaryngol Clin N Am 37:1209-1228

Taylor SE, Brown J (1988) Illusion and well-being: a social psychological perspective on mental health. Psychol Bull 103:193210

Wehling E, Nordin S, Espeseth T, Reinvang I, Lundervold A (2011) Unawareness of olfactory dysfunction and its association with cognitive functioning in middle aged and old adults. Arch Clin Neuropsychol 26:260-269

Weinstein ND (1980) Unrealistic optimism about future life events. J Pers Soc Psychol 39:806-820

Welge-Luessen A, Hummel T, Stoja T, Wolfensberger M (2005) What is the correlation between ratings and measures of olfactory function in patients with olfactory loss? Am J Rhinol 19:567571

White TL, Kurtz DB (2003) The relationship between metacognitive awareness of olfactory ability and age in people reporting chemosensory disturbances. Am J Psychol 116:99110

Wood JV (1989) Theory and research concerning social comparison of personal attributes. Psychol Bull 106:231-248 